\title{
How is place of death from cancer changing and what affects it? Analysis of cancer registration and service data
}

\author{
E Davies*,', KM Linklater', RH Jack', L Clark' and H Møller' \\ 'King's College London, School of Medicine at Guy's, King's and St Thomas' Hospitals, Thames Cancer Registry, Capital House, 42 Weston Street, London \\ SEI 3QD, UK
}

\begin{abstract}
We aimed to compare trends in place of cancer death with the growth of palliative care and nursing home services, and investigate demographic, disease-related and area influences on individual place of death, using registration data for 216404 patients with breast, lung, colorectal and prostate cancer and aggregate data on services in South East England. Between 1985 and 1994 there was a trend away from hospital death (67-44\%), to home (17-30\%) and hospice death (8-20\%). After 1995, this partly reversed. By 2002, hospital death rose to $47 \%$, home death dropped to $23 \%$, hospice death remained stable and nursing home death rose from 3 to $8 \%$. Numbers of palliative care services increased, but trends for hospice and nursing home deaths most clearly followed the beds available. Cancer diagnosis and treatment influenced individual place of death, but between 1998 and 2002, age and area of residence were associated with most variation. Older patients and those living in more deprived areas died more often in hospitals and less often at home. Despite more palliative care services the proportion of people dying at home has not increased. Variation by age, deprivation and area of residence is unlikely to reflect patient preference. More active surveillance and planning must support policies for choice in end of life care.
\end{abstract}

British Journal of Cancer (2006) 95, 593-600. doi: I 0.1038/sj.bjc.6603305 www.bjcancer.com

Published online 15 August 2006

(C) 2006 Cancer Research UK

Keywords: palliative care; place of death; cancer registration; health services researches

Most people say they would prefer to die at home, but in reality most patients in the UK spend their final days in hospital (WHO, 2004a). Over the last 30 years, hospices, palliative care teams and units have developed with the aim of improving care towards the end of life, and allowing people to die where they wish, if this is possible. Services have initially focused on patients with cancer, primarily because of the relative ease of predicting the course of this disease, and a national policy for supportive and palliative cancer care is now in place (House of Commons Health Committee, 2004; NICE, 2004a, b). There is, therefore, increasing interest in Europe about whether data on place of death can be used as an interim measure of the success of services provided (WHO, 2004a). Figures for England and Wales revealed a trend away from death in hospital or nursing homes to hospices between 1985 and 1994, but very little change in home deaths, which remained around $26 \%$. However, this proportion varied between regions and was lowest in South East England across all age and cancer types (Higginson et al, 1998). For common cancers, individual, disease-related and area of residence factors were consistently associated with, but not strongly predictive of place of death. Men, patients aged under 74 years, those with lung or colorectal cancer or living in more affluent areas were more likely to die at home than women, patients aged over 75 years, those with breast cancer or those living in less affluent areas (Higginson et al, 1998, 1999).

*Correspondence: Dr E Davies E-mail: Elizabeth.Davies@kcl.ac.uk Received 30 May 2006; accepted 7 July 2006; published online 15 August 2006
The Thames Cancer Registry covers a population of 14 million people in South East England, an area with one of the highest concentrations of hospice and palliative care services in the UK (Hospice Information, 2006). We used Registry data to describe trends in place of death for common cancers and compared these to the growth of palliative care services and nursing homes between 1985 and 2002. We then investigated the relationship between demographic, disease-related factors and individual place of death throughout the period, and the additional influence of area of residence between 1998 and 2002.

\section{METHODS}

In the UK cancer registries record the occurrence of cancer in their residential populations as well as treatments given in the first 6 months after diagnosis. Information about death is provided by the National Health Service Central Register through the Office for National Statistics. Death certificates routinely record place of death and assign cancer as a main or contributing cause of death in part I of the certificate.

We extracted data on 216404 residents in South East England who had been diagnosed with breast, lung, colorectal and prostate cancer between 1985 and 2002, and who died from their disease between 1985 and 2002. Cases where the only registration information was from the death certificate were not included. From death certificates, we classified death as occurring in NHS acute hospitals, hospices, long stay hospitals or nursing homes, private hospitals, at home or as unknown. We could identify nursing homes by their address, but death certificates do not 
distinguish deaths in hospital palliative care units from those in other wards.

We extracted data on hospice and palliative care services from Hospice Information directories for 1985-2002 (Hospice Information, 2006) and calculated the number of hospice beds, home care teams, day care services, hospital palliative care or support teams and hospital support nurses in our area. We summed home care services regardless of their funding (independent, NHS and Macmillan Cancer Relief) or base (hospices, NHS hospital or community), although there was insufficient detail about Marie Curie home services to include these. We could not deduce team size or caseload. We also obtained aggregate data on numbers of beds in registered nursing homes from the Department of Health where this was available for 1991-2001. We first plotted the proportion of deaths occurring in each of home, hospital, hospice and nursing home against the growth of different services over time. Data on acute and general hospital beds in our area were available only between 1996 and 2002 and were not plotted.

We then took death in hospice, nursing home, NHS acute hospital and at home as our four dependent variables and fitted logistic regression models to identify individual demographic, disease-related and area of residence factors predicting in turn each of these outcomes versus the others. Our first analysis for the entire period 1985-2002 included sex, age at diagnosis, whether the diagnosis was based on clinical or microscopic evidence, primary site of cancer and treatment with surgery, radiotherapy, chemotherapy or hormone therapy. We also adjusted for year of death and years since diagnosis to examine trends over time. We grouped age into four bands: $<65$ years, $65-74$ years, $75-84$ years and 85 years plus. Our second analysis explored the additional influence of area of residence for the years 1998-2002. For this, we assigned each individual to an electoral ward and a cancer network using their postcode of residence. We calculated the deprivation score for each ward using the income domain of the Indices of Multiple Deprivation (IMD) 2000 for England (Department of Environment, 2000) and assigned individuals to a quintile of deprivation ranging from most (1) to least affluent (5) wards.

We present the results of logistic regression analyses as proportions of deaths occurring in each place for each factor. Proportions are easier to interpret than odds ratios, and were derived from a back calculation from the odds ratios obtained from the logistic regression analyses. We present unadjusted and adjusted proportions to show the effect of controlling for all other factors. Our large sample size means that many small differences reach statistical significance. We draw attention only to those factors producing at least five percentage points difference - a difference which we believe a clinical service might be interested to explore further.

\section{RESULTS}

The average age of death for patients in this cohort increased from 71.3 years in 1985 to 72.7 in 2002 . The proportion dying at age of 85 years and over increased from 8 to $12 \%$ while the proportion dying between the age of 65 and 74 years dropped from 34 to $28 \%$.

\section{How has place of death changed?}

Figure 1 suggests that the period 1985-2002 is best considered in two phases - before and after 1994. In the first phase, hospital deaths declined from 67 to $44 \%$ - a trend that appeared to be mirrored by a combined increase in home death from 17 to $30 \%$ and in hospice death from 8 to $20 \%$. In the second phase, however, the movement away from hospital death appeared to partly reverse. Between 1995 and 2002 hospital death rose to $47 \%$, nursing home death to $8 \%$, hospice death remained stable and home deaths dropped to $23 \%$. In 2002 - the last year of the study -

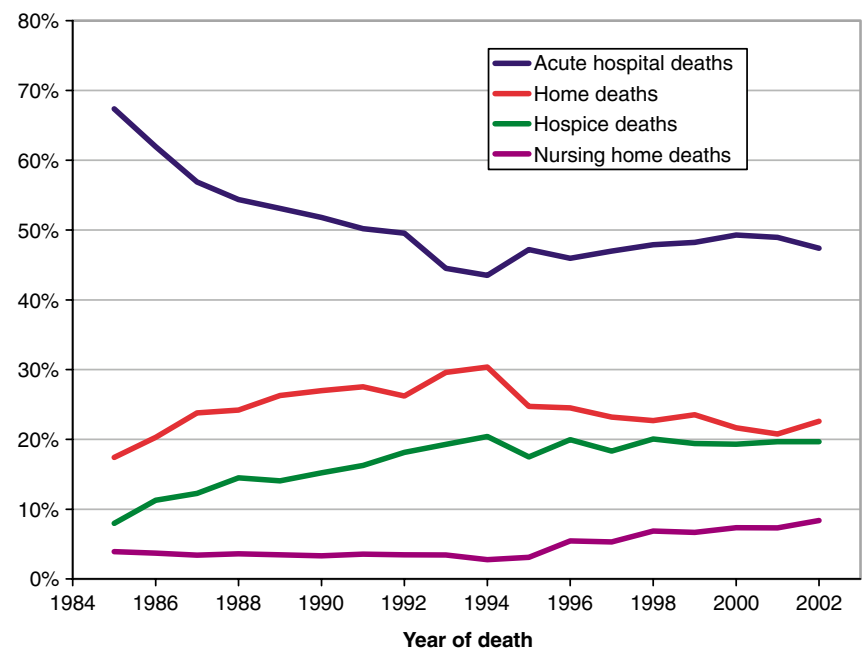

Figure I Trends for place of death for patients with breast, colorectal, lung and prostate cancer in South East England 1985-2002. Note: Figure excludes the proportion dying in private hospitals and patients where place of death was not known.

home death and nursing home death home appear to have increased slightly and hospital death to have decreased. During 1992 and 1995 there were changes in processing and receipt of our registry data which may be responsible for the 'mirroring' of trends in hospital and home deaths during this period. This artefact overlies but does not explain the reversal of overall trends which is also seen in national data for this period.

Figure 2 shows that during the first phase, while home and hospice death increased, the provision of home care services and hospice beds also increased. From 1995 onwards while nursing home death and hospital death increased, nursing home beds and, to a lesser degree, the sum of hospital palliative care services (teams and nurses) also increased. For nursing home deaths, unlike hospice deaths, there is a lag of several years between the rise of available beds in these services and deaths within them. The decline in home death occurring after 1995 did not appear to follow a substantial drop in the provision of palliative home care or day care services, which both remained stable, although during this period the availability of nursing home beds was increasing.

\section{Which individual and disease-related factors affect place of death?}

Table 1 shows unadjusted and adjusted proportions of deaths in each place for individual demographic and disease-related factor over the entire study period. Hospital death was more likely for patients aged over 75 , those with lung or breast cancer, a clinical rather than microscopic diagnosis, and those not receiving radiotherapy. Home death was more likely for those with colorectal cancer and those aged less than 75 years. Hospice death was also more likely for colorectal cancer and for those aged less than 75 years. Nursing home death increased with older age (4\% for those aged $65-74$ years and $12 \%$ of those aged over 85 years).

\section{Did place of residence affect place of death between 1998 and 2002?}

Our analysis for the most recent years included area of residence as assessed by cancer network of residence and deprivation of ward of residence (Table 2). The results for demographic and diseaserelated factors were broadly similar to those in Table 1, although nursing home deaths become more likely for those with breast and prostate cancer. However, much more striking was the variation by 

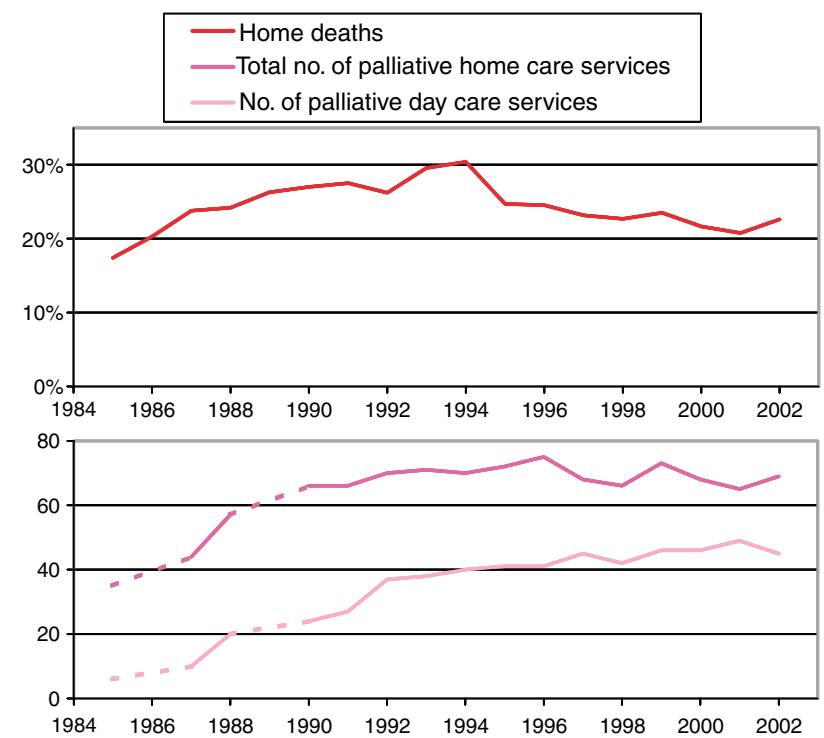

$\begin{array}{llllllllll}1984 & 1986 & 1988 & 1990 & 1992 & 1994 & 1996 & 1998 & 2000 & 2002\end{array}$
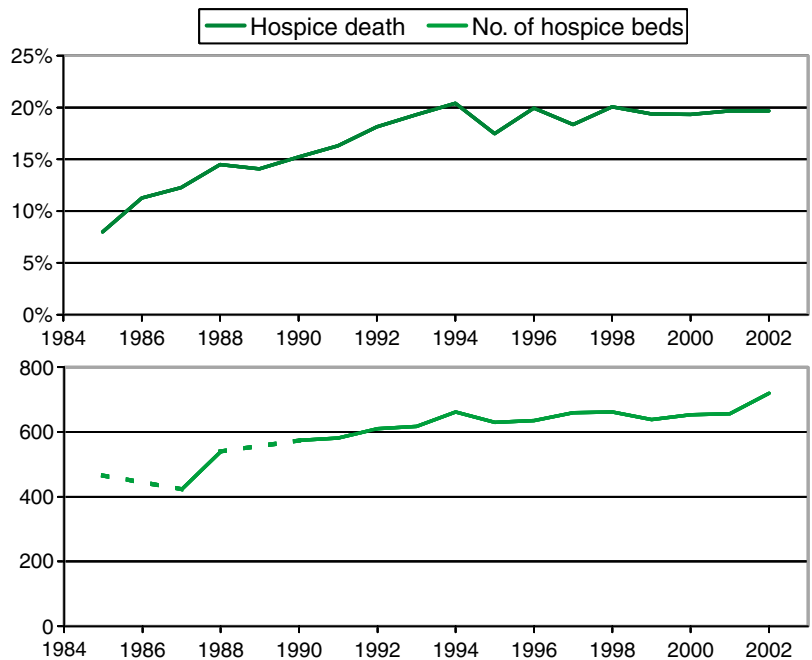
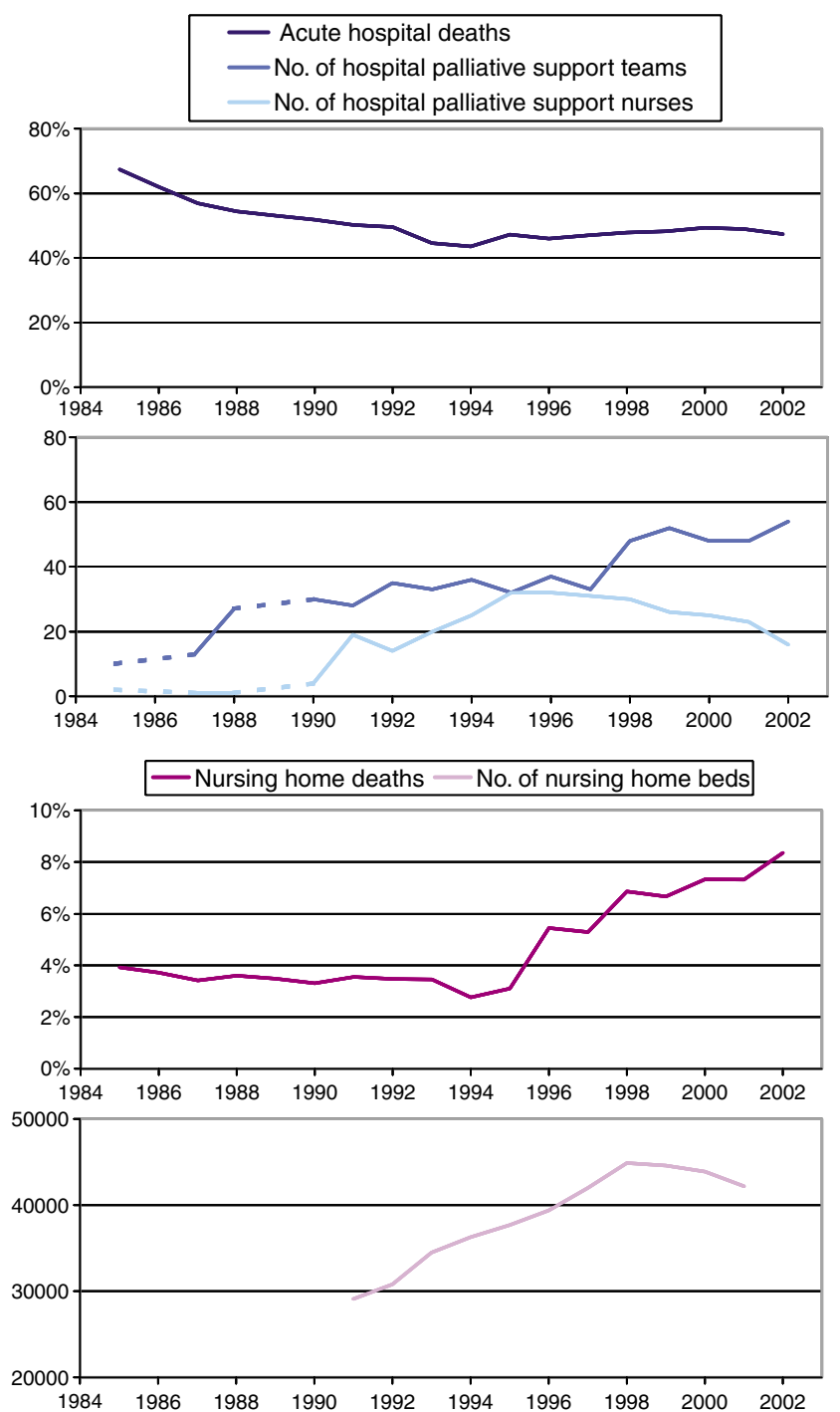

Year of death

Figure 2 Trends in place of death for patients with breast, lung, colorectal and prostate cancer and the growth of services for care towards the end of life in South East England 1985-2002. Note: Department of Health Data is only available for I99 I-200 I and Hospice Information Directories are not available for 1986 and 1989.

area of residence. Concentrating on the nine of 13 cancer networks that we completely cover, the adjusted proportion of patients dying in hospital ranged from $39 \%$ in Sussex to $60 \%$ in West London. Home deaths ranged from $16 \%$ in Surrey, West Sussex and Hampshire to $27 \%$ in South Essex. Hospice death ranged from $10 \%$ in West London to $31 \%$ in Surrey, West Sussex and Hampshire. Nursing home deaths ranged from $4 \%$ in North London to $13 \%$ in Sussex. Of London networks, South East London had the lowest rate of hospital death $(49 \%)$ and the highest rate of home death (23\%). Patients from more deprived areas died more often in hospital and less often at home. There was no important deprivation gradient for nursing home or hospice death.

\section{DISCUSSION}

\section{Summary of main findings}

This study of 216404 patients diagnosed and dying from four common cancers in South East England found an initial trend away from hospital death $(67-44 \%)$ to home (17-30\%) and hospice death $(8-20 \%)$ between 1985 and 1994. After 1995 this trend partly reversed. By 2002, the proportion of hospital deaths rose to $47 \%$, hospice deaths remained stable, home deaths dropped to $23 \%$ and nursing home deaths rose from 3 to $8 \%$. The number and range of palliative care services increased but trends for hospice and nursing home death most clearly followed the numbers of beds available. Analysis of individual data showed that throughout the period disease-related factors had a modest influence on place of death. Patients with colorectal cancer were more likely to die at home and in hospices while patients with lung or breast cancer, no microscopic diagnosis and no radiotherapy were more likely to die in hospitals. However, between 1998 and 2002, age and place of residence were associated with most variation. Older patients were more likely to die in hospitals and nursing homes and less likely to die at home or in hospices. Patients from deprived areas were more likely to die in hospitals and less likely to die at home. There was significant variation in each place of death by cancer network of residence. 
Table I Associations of individual demographic and disease-related factors with place of death for cancer patients who died $1985-2002$ in South East England

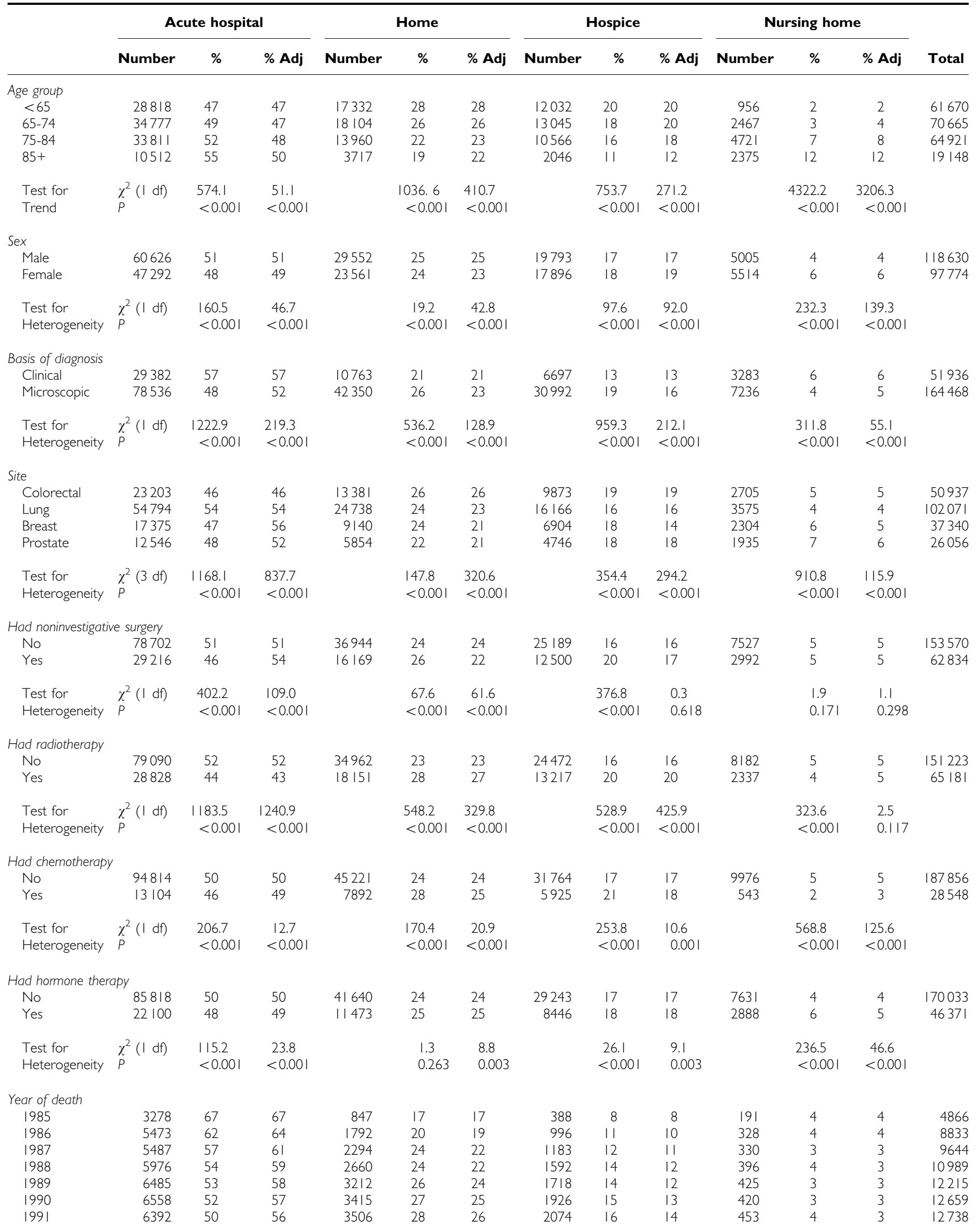


Table I (Continued)

\begin{tabular}{|c|c|c|c|c|c|c|c|c|c|c|c|c|c|}
\hline & \multicolumn{3}{|c|}{ Acute hospital } & \multicolumn{3}{|c|}{ Home } & \multicolumn{3}{|c|}{ Hospice } & \multicolumn{3}{|c|}{ Nursing home } & \multirow[b]{2}{*}{ Total } \\
\hline & Number & $\%$ & $\%$ Adj & Number & $\%$ & $\%$ Adj & Number & $\%$ & $\%$ Adj & Number & $\%$ & $\%$ Adj & \\
\hline 1992 & 6274 & 50 & 55 & 3318 & 26 & 24 & 2296 & 18 & 16 & 440 & 3 & 3 & 12660 \\
\hline 1993 & 6536 & 45 & 50 & 4345 & 30 & 28 & 2834 & 19 & 17 & 507 & 3 & 3 & $1468 \mid$ \\
\hline 1994 & 5342 & 44 & 50 & 3730 & 30 & 28 & 2505 & 20 & 17 & 339 & 3 & 2 & 12279 \\
\hline 1995 & 6055 & 47 & 54 & 3170 & 25 & 22 & 2242 & 17 & 15 & 398 & 3 & 2 & 12827 \\
\hline 1996 & 4917 & 46 & 53 & 2625 & 25 & 22 & 2135 & 20 & 17 & 583 & 5 & 5 & 10704 \\
\hline 1997 & 5606 & 47 & 54 & 2765 & 23 & 21 & 2188 & 18 & 15 & 631 & 5 & 4 & 11930 \\
\hline 1998 & 6463 & 48 & 55 & 3062 & 23 & 20 & 2709 & 20 & 17 & 927 & 7 & 6 & $1350 \mid$ \\
\hline 1999 & 6653 & 48 & 55 & 3247 & 24 & 21 & 2675 & 19 & 17 & 920 & 7 & 5 & 13798 \\
\hline 2000 & 6849 & 49 & 56 & 3008 & 22 & 20 & 2685 & 19 & 17 & 1019 & 7 & 6 & 13893 \\
\hline 2001 & 6812 & 49 & 55 & 2892 & 21 & 19 & 2737 & 20 & 17 & 1019 & 7 & 6 & 13919 \\
\hline 2002 & 6762 & 47 & 54 & 3225 & 23 & 21 & 2806 & 20 & 17 & 1193 & 8 & 7 & 14268 \\
\hline Test for & $\chi^{2}(I d f)$ & 906.0 & 353.0 & & 68.3 & 119.2 & & 949.9 & 672.1 & & 1087.3 & 648.8 & \\
\hline Trend & $P$ & $<0.001$ & $<0.001$ & & $<0.001$ & $<0.001$ & & $<0.001$ & $<0.001$ & & $<0.001$ & $<0.001$ & \\
\hline
\end{tabular}

Adjusted model includes: age, sex, basis of diagnosis, site, treatment (surgery, radiotherapy, chemotherapy or hormone therapy), year of death and years since diagnosis.

Table 2 Associations of individual demographic, disease-related and area of residence with place of death for patients who died from Breast, lung, colorectal or prostate cancer between 1998 and 2002 in South East England

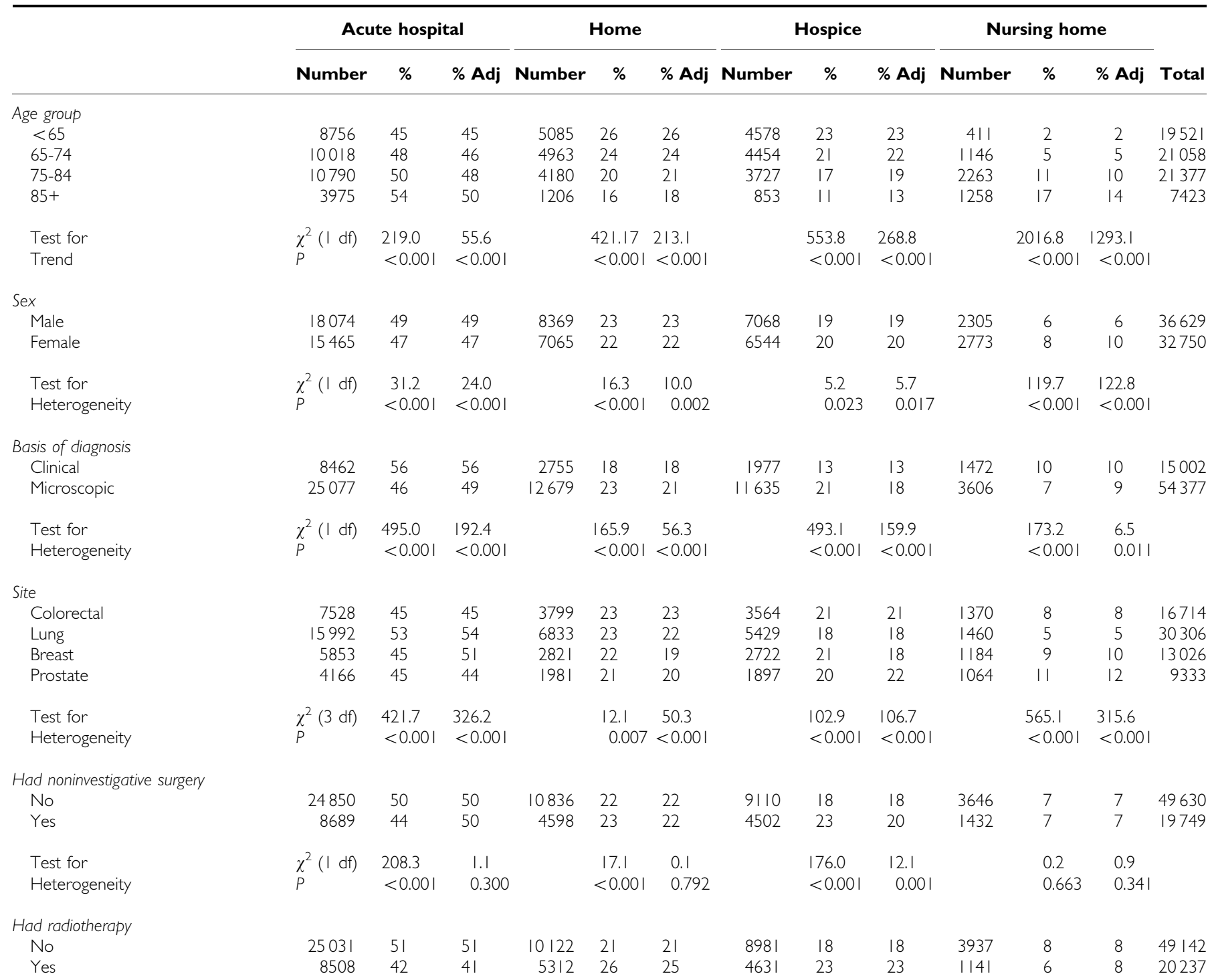


Table 2 (Continued)

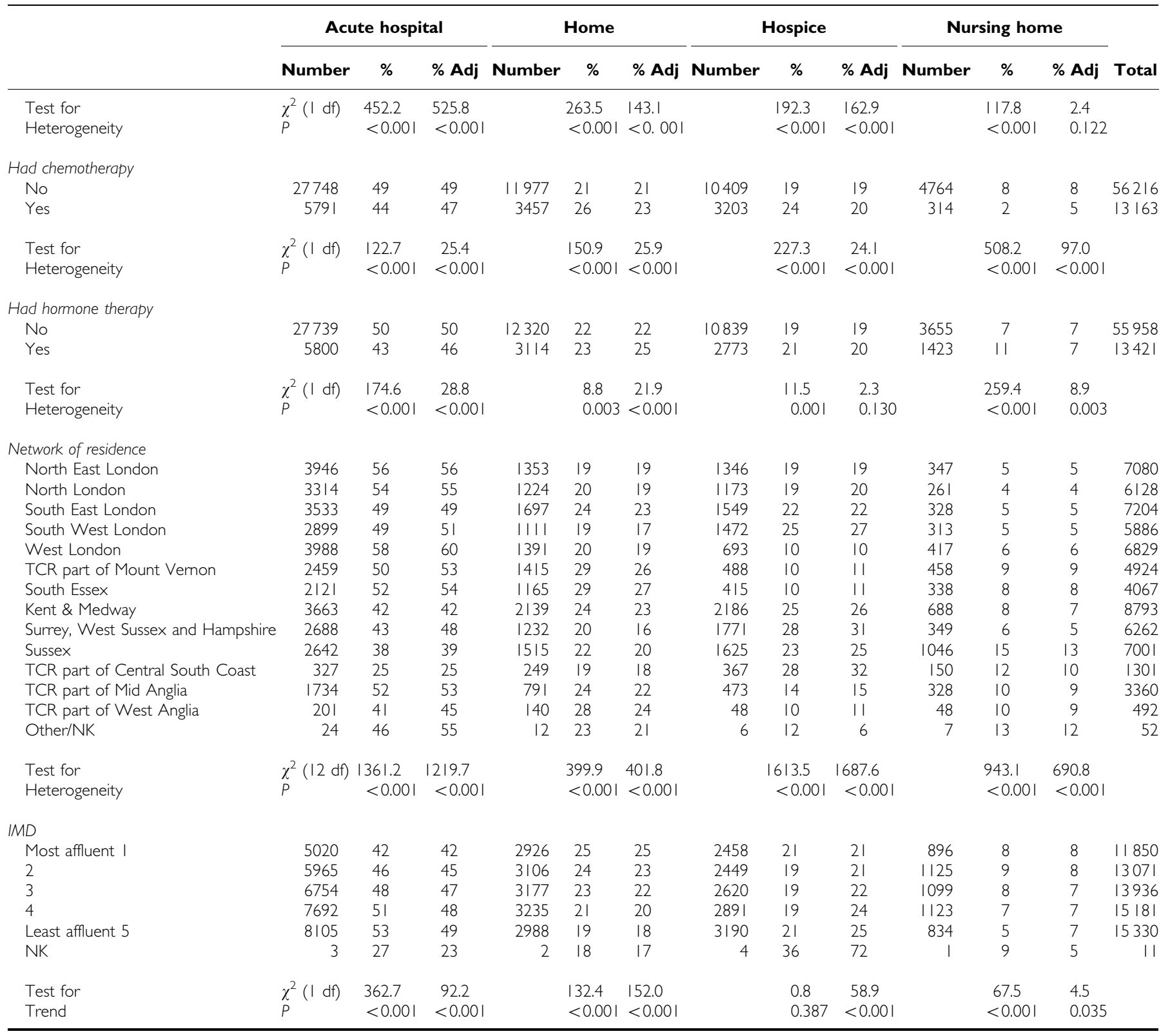

Adjusted model includes: age, sex, basis of diagnosis, site, treatment (surgery, radiotherapy, chemotherapy or hormone therapy), cancer network of residence and deprivation.

\section{Limitations of this study}

This population-based study used data collected from medical records and death certificates for routine cancer registration. Coding officers may have missed some deaths in new nursing homes and hospices when their addresses were unfamiliar in the early part of the study period. Lack of information on death certificate on deaths in hospital palliative care units and lack of data on hospital beds meant we could not explore these trends and it is possible that excluding patients for whom we had only death certificate data from the analysis introduced some bias. Important information on patient preference for place of death, functional status, presence of a carer at home, family support, and hospital and community services received in the weeks before death (Grundy et al, 2004; Gomes and Higginson, 2006) is not routinely collected and is therefore missing from the individual analyses.

\section{Comparison to other findings}

No other large UK studies have compared overall trends in place of death with the growth of services that might support patients to die in different places. However, one study of North West England between 1993 and 2000 found that proximity to a hospice or hospital increased the chances of dying there (Gatrell et al, 2003). Studies in the US have also found that the availability of beds and physicians affects death in hospital (Fisher et al, 2003a,b). National bed data available for 1987 to 1994 when hospital deaths decreased showed a decline of $19 \%$ in the numbers of acute and general hospital beds (Department of Health, 2006). It, therefore, seems likely that the initial trend for increasing home death was in part driven by the decreased availability of hospital beds and the growth of hospice and palliative home care services. However, it is more difficult to explain the reversal of the trend for home death 
after 1995 using the routine service data that is available. The number of home care teams did not decline, hospital palliative care services were only just beginning to increase and hospital beds did not increase nationally until 2001. We can speculate that the decline in home death was due to other changes in care at home including the ability of families to provide care, the prior move of some older adults into nursing homes and the move to GP cooperatives for out of hours care. These factors could all have led to increased hospital admission and fewer home deaths.

Turning to predictors of individual place of death, our finding that younger patients, patients with colorectal cancer and those living in more affluent areas died more often at home is consistent with (Higginson et al 1998, 1999) analyses of a partial national registration dataset up until 1994 . However, we found that patients with breast and lung cancer were more likely to die in hospital and we were further able to show that hospital death was associated with lack of microscopic diagnosis, and no radiotherapy treatment. This suggests the late admission of patients with advanced stage of disease. Conversely our finding that a microscopically confirmed diagnosis and radiotherapy treatment were associated with home and hospice death suggests that some time within 'the system' may allow for referral to supportive services (Burge et al, 2003). A recent systematic review of factors predicting home death by Gomes and Higginson (2006) found that the six strongest predictors were patients' low functional status, their preferences, home care and its intensity, living with relatives and extended family support. Our new finding that cancer network is an important cause of variation in home death is consistent with this, and probably represents a combination of difference by area in access to home care services, and the nearness of relatives and extended family. It is very unlikely to represent underlying variation in patients' preference for place of death or functional status. Finally our finding that patients from more deprived areas were equally likely to die in hospices and nursing homes as those from affluent areas, contradicts the view that the latter may access these services more often. Inequalities in hospital and home death do, however, persist.

\section{Implications for practice and policy}

Our findings reveal that despite increased investment in and provision of palliative care services, cancer patients in South East England remain twice as likely to die in hospital (47\%) than at home $(23 \%)$. The proportion dying at home is now lower than a decade ago, lower than elsewhere in the UK, and far lower than most patients would prefer. Recent national policy has set out the evidence that coordinated palliative care services can allow more people to die at home if they wish (NICE, 2004a, b) and advocated equity of choice in final place of care. This study covers a period before most recent initiatives (Gold Standards Framework, 2006; Marie Curie, 2006) but the variation it finds underlines the need for much more active local surveillance to drive these policies. It also suggests that opportunities exist to learn from differing strategies, organisation and practice within cancer networks. For example, London networks might ask what it is about service provision in South East London that produces rates of home death similar to those outside London. Networks outside London might ask why hospice deaths are sometimes so high and whether nursing homes are preventing hospital admission and providing better symptom control. Our data also suggest that a good place for clinicians in primary care and acute trusts to start identifying patients in the palliative stage of disease and determining their preference for avoiding or planning admission would be the clinical diagnosis of lung or breast cancer in patients living in deprived areas for whom radiotherapy treatment is not planned. The effect of any change in practice across a network can be monitored easily by the routine work of cancer registries.

\section{Further research}

We do not yet fully understand why place of death varies across the UK, how the nexus of factors around the patient operate together to influence this (Gomes and Higginson, 2006) and why home deaths have declined and remain so low in South East England. The imaginative use of available routine data as part of the development of cancer intelligence could help us see more clearly what is happening. For example, trends within individual cancer networks could reveal the influence of different historical patterns of service provision. Ecological studies could show us what happens when a new service such as a hospice opens locally. Mapping rates geographically by primary care trust could show the influence of services (beds and teams) and workforce (district nurses (Shipman et al, 2005), Marie Curie nurses and out of hours care by general practitioners). Studies of how patients move between different services and the interdependence between services are also required. Linking hospital episode statistics data with cancer registration data will, for example, allow us to explore where patients with different cancers are admitted to hospital from, how long they stay and where they are discharged to in the last months of life. The influence that admission has on rate of death in different trusts or primary care trusts explored in a similar way to US studies have done (Wennberg et al, 2004). Qualitative case studies of selected areas could then focus on explaining how different patterns of care are perpetuated, or how change has occurred. Finally in the context of an ageing population, changes in migration and kinship patterns we need to determine older people's preference for death in institutions, ensure that the information we have on what currently occurs is available and public so that where possible people may make their own choices in planning care towards the end of life (WHO, 2004b).

\section{ACKNOWLEDGEMENTS}

We thank Hospice Information for providing us with Hospice Directories, the Department of Health for confirming the nursing home data and colleagues for helpful comments on an earlier draft of this paper.

\section{REFERENCES}

Burge F, Lawson B, Johnston G (2003) Trends in the place of death of cancer patients. Can Med Assoc J 163(3): 265-270

Department of the Environment, Transport and the Regions (2000) Indices of Deprivation 2000: Regeneration Research Summary No. 31. London: Stationery Office.

Department of Health (2006) Hospital Activity Statistics. 1987-2005, www.performance.doh.gov.uk/hospitalactivity/data_requests/download/ beds_open_overnight/beds_ts.xls (Accessed 17th March 2006)
Higginson IJ, Astin P, Dolan S (1998) Where do cancer patients die? Tenyear trends in the place of death of cancer patients in England. Palliative Med 12: $353-363$

Higginson IJ, Jarman B, Astin P, Dolan S (1999) Do social factors affect where patients die: an analysis of 10 years of cancer deaths in England. J Public Health Med 21(1): $22-28$

Hospice Information Service (2006) http://www.hospiceinformation.info/ (Accessed 10th March 2006) 
House of Commons Health Committee (2004) Palliative Care. Fourth report of the session 2003-4. London: Stationary Office

Fisher ES, Wennberg DE, Stukel DA, Gottleib D, Lucas FL, Pinder E (2003a) The implications of regional variations in Medicare spending: part 1, utilisation of services and quality of care. Ann Intern Med 138: 273 -287

Fisher ES, Wennberg DE, Stukel DA, Gottleib D, Lucas FL, Pinder E (2003b) The implications of regional variations in Medicare spending: part 2, health outcomes and satisfaction with care. Ann Intern Med 138: $288-298$

Gatrell AC, Harman JC, Francis BJ, Thomas C, Morris SM, Mclllmurray M (2003) Place of death: analysis of cancer deaths in North West England. J Public Health Med 25(1): $53-58$

Gold Standards Framework (2006) http://www.goldstandardsframework. nhs.uk/ (Accessed 17th March 2006)

Gomes B, Higginson IJ (2006) Factors affecting death at home in terminally ill patients with cancer: systematic review. $\mathrm{Br}$ Med J 332: 515-521

Grundy E, Mayer D, Young H, Sloggett A (2004) Living arrangements and place of death of older people with cancer in England and Wales: a record linkage study. Br J Cancer 91: 907-912
Marie Curie (2006) Liverpool Care of the Dying Pathway. http://www. lcp-mariecurie.org.uk/Accessed 10th March 2006

National Institute for Clinical Excellence (2004a) Guidance on Cancer Services Improving Supportive and Palliative Care for Adults with Cancer. The Research Evidence. London: National Health Service

National Institute for Clinical Excellence (2004b) Guidance on Cancer Services: Improving Supportive and Palliative Care for Adults with Cancer. The Manual. London: National Health Service

Shipman C, Addington-Hall J, Richardson A, Burt J, Ream E, Beynon T (2005) Palliative care services in England: a survey of district nurses' views. Br J Com Nurs 10(8): $381-386$

Wennberg JE, Fisher ES, Stukel TA, Skinner JS, Sharp SM, Bronner KK (2004) Use of hospitals, physician visits, and hospice care during the last six months of life among cohorts loyal to highly respected hospitals in the United States. Br Med J 328: 607-612

WHO Regional Office for Europe (2004a) Palliative Care - The Solid Facts. Copenhagen

WHO Regional Office for Europe (2004b) Better Palliative care for older people. Copenhagen 